\title{
81. FORMATION OF COMETS IN METEOR STREAMS
}

\author{
J. TRULSEN
}

The Auroral Observatory, University of Tromsø, Tromsø, Norway

\begin{abstract}
The temporary focusing effect of planetary perturbations on the particles of a meteor stream is studied. It is indicated that allowance for collisions could result in more permanent focusing, leading to the possible formation of a comet.
\end{abstract}

The present work was started following a suggestion by Alfvén, who has recently stressed the possible importance of material streams in the processes leading to the formation of the solar system. It was felt that the traditional theories for the formation of short-period comets, both the capture theory and the eruption theory, were not completely satisfactory. The capture theory has been discussed by a number of authors, most recently in a series of papers by Everhart (1967a, 1967b, 1968, 1969), whereas Vsekhsvyatskij (1966) has worked out arguments in favour of eruption. By short-period comets we shall mean here comets with periods less than or about that of Jupiter.

It is well known that several comets are associated with meteor streams. The question was therefore posed as to whether the short-period comets could in fact be formed out of meteor streams. The existence of a large number of such streams out in space is readily inferred from observed meteor showers on the Earth (Lindblad, 1971). In order to substantiate the above picture it was necessary to find a mechanism that could focus a considerable fraction of the material dispersed in the stream into a smaller region and then prevent this material from subsequent spreading. The above high density region would then constitute the new-born comet, possibly existing in a kind of equilibrium with the remnants of the meteor stream. I should like to discuss here the possible initial phase of this creation process - the focusing of the material in the meteor stream.

Similar situations are well known in plasma physics. If, for example, an electron beam passes through a region where it is subject to a varying electric field, there is often observed bunching of the electrons after they have emerged from the perturbing region. Processes of this kind are indeed of great importance in the electronics industry.

In the present case the electron beam is, of course, replaced by the meteor stream, and it was thought that the necessary perturbations could be produced by Jupiter through a close approach to the stream. In order to study the problem quantitatively the following simple model was considered. A tenuous meteor stream is moving around a central body around which there is also a planet in a circular orbit. The mass ratio between the planet and the central body is chosen to correspond to that of the Jupiter-Sun system. In order to reduce the number of parameters involved the meteor stream and the planet are assumed to have the same orbital plane. Preliminary studies indicate that there is no qualitative difference between the results of this two-dimensional system and those of the more general case, except for the fact that the chance of a 
real close approach of part of the meteor stream to the planet is larger in the former case.

A group of particles in the stream is now selected, and the focusing or spreading of this group of particles relative to the unperturbed stream is studied numerically. The group selected consists of particles originally following the same elliptical orbit (but they are displaced slightly along that orbit on account of the perturbations by the planet). To specify the different groups of particles a rotating coordinate system was used, in which the planet is the origin and the central body is on the negative $x$-axis. The parameters chosen to identify each group are the position of the aphelion of the central particle in the group in this coordinate system and the eccentricity of the orbit at the time of aphelion passage.

Figure 1 illustrates some typical examples of the rms distance between particles for an eccentricity $e=0.4$, for three different aphelion distances and for six different rotations of the aphelion relative to the planet. As is easily seen, the focusing of the meteor stream by the planetary perturbations represents an appreciable effect. Reduction in intermeteoric distance by one order of magnitude relative to the unperturbed value is quite common. The maximum focusing achieved in the results presented here amounts to a factor of more than 20 .

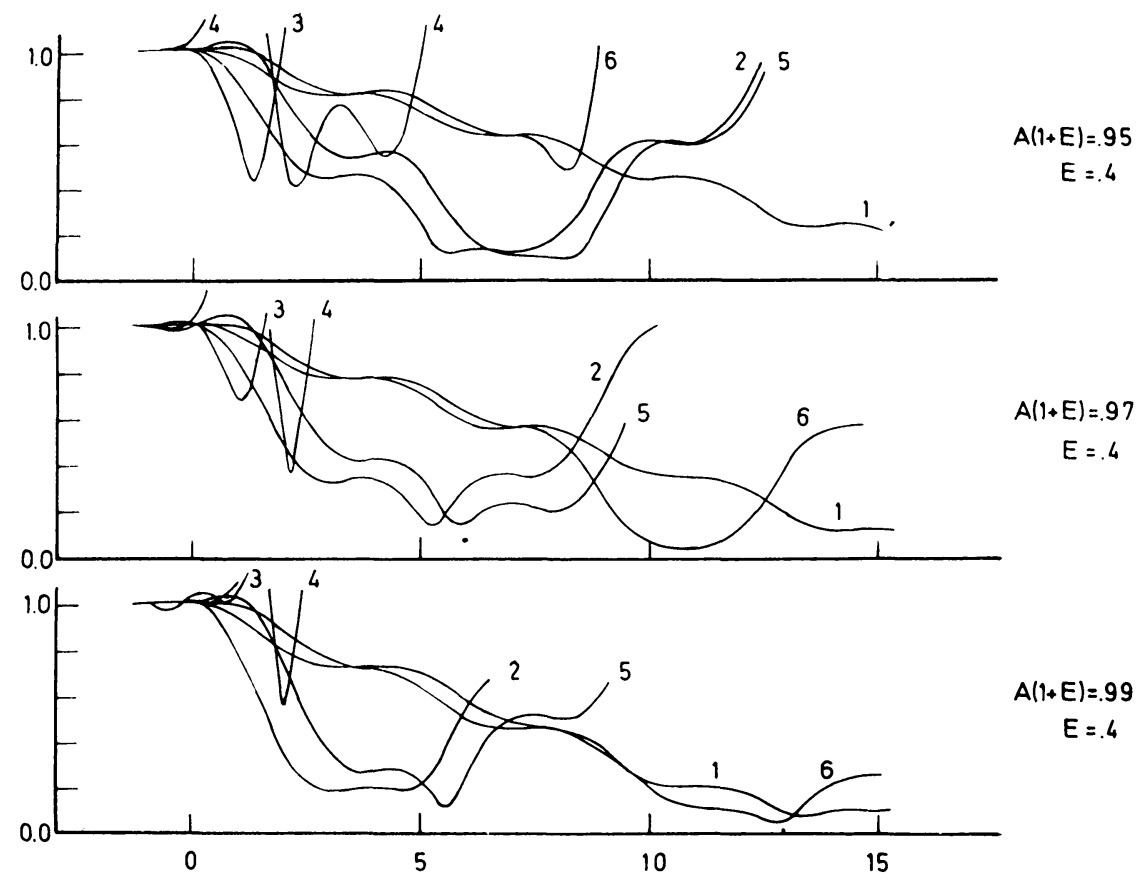

Fig. 1. The rms distance between particles in each group, normalized so that in the absence of planetary perturbations the ordinate would be unity (i.e., the density variations in the stream due to the variable velocity in the elliptical orbit are taken out). The curves are for eccentricity $e=0.4$ and for aphelion distances $a(1+e)=0.95,0.97$, and 0.99 (the distance between the Sun and the planet being unity). The curves, labelled $1 \cdots 6$, refer to the cases where the angle planet-Sun-aphelion is equal to $-30,-20,-10,+10,+20$, and +30 , respectively. 
The physical mechanism behind the focusing is very similar to the electron beam experiment referred to above. In order to achieve any focusing the particles in each group must be subject to a velocity modulation. In the case studied here this takes place around aphelion, when the distance meteor-planet is smallest. There is thus no effect before aphelion passage. After the velocity modulation has taken place the system needs some time before the maximum effect is achieved - the longer the time the smaller the modulation (see curves 1 and 6 in Figure 1). The modulation should not be too large, however (curves 3 and 4); in this case what is observed is rather the scattering of the group of particles. In this respect there is an important difference between the present theory for the origin of short-period comets and both the capture and the eruption theories. In our case the perturbations by Jupiter should not exceed a certain level. The mechanism works when the minimum distance between the particles and Jupiter is of the order of $1 \mathrm{AU}$. The calculations indicate that the maximum focusing achieved is relatively insensitive to the strength of the perturbations and that it is mainly the focusing time that varies.

It is possible to give a simple physical description of the resulting perturbations in the stream. To this end let us pick as a reference the particular meteor that in the rotating coordinate system has an orbit symmetrical about the planet-Sun line. The perturbations imposed on the stream may now be regarded as a density wave propagating along the stream in both directions from this reference meteor. For the parameters chosen here disruption of the stream takes place in the region adjacent to the reference meteor. Farther out, however, a density increment is gradually built up with a maximum amplitude about one order of magnitude above the unperturbed level. The wave crest then passes outwards and the group of particles spreads out again. This dispersion is discussed by Levin et al. (1972). For our purpose this eventual dispersion is of no interest. The important thing to note is that an appreciable focusing is produced, and that for the Jupiter-Sun system its duration is measured in years.

The work described here necessarily represents only a preliminary study of the model. Only the possible initial phase of this creation process has been discussed since the above mechanism can only produce a temporary focusing. To arrive at a more permanent focusing viscous effects have to be included - these being in the form of collisions between the meteors themselves and possibly also between meteors and released gas. These are highly nonlinear effects, and we should expect that there exists a limiting density above which such effects become of crucial importance, possibly in the way that the above density wave is arrested in a certain element of the stream. One possible way of achieving this critical density may be through interference between two such waves excited at consecutive close approaches to the planet. A study of viscous effects on the focusing is underway. It is hoped that this study will give insight into the conditions that must be satisfied for the present model to work. We might then have a better possibility to judge on the probability of this model even if very little is known of the conditions in a pre-comet meteor stream.

In trying to find connections between the present model and the first-apparition short-period comets two factors are of importance: the position of the pre-apparition orbits of the comets relative to Jupiter, and the time of first apparition. The latter 
factor is important because we should allow enough time for the focusing to develop. A preliminary study of this question has been undertaken, and it shows that for the majority of the first-apparition short-period comets these factors are indeed in favour of the present model.

\section{References}

Everhart, E.: 1967a, Astron. J. 72, 716.

Everhart, E.: 1967b, Astron. J. 72, 1002.

Everhart, E.: 1968, Astron. J. 73, 1039.

Everhart, E.: 1969, Astron. J. 74, 735.

Levin, B. Yu., Simonenko, A. N., and Sherbaum, L. M.: 1972, this Symposium, p. 454.

Lindblad, B. A.: 1971, Smithsonian Contr. Astrophys. 12.

Vsekhsvyatskij, S. K.: 1966, Mem. Soc. Roy. Sci. Liège Ser. 5 12, 495. 